\title{
Implementación de un Modelo de un Transformador Eléctrico para el Análisis de su Respuesta en Frecuencia
}

\author{
Guillermo Aponte $^{(1)}$, Wilder Herrera ${ }^{(1)}$, Carlos González $^{(2)}$, Jorge Pleite $^{(2)}$ \\ (1) Universidad del Valle, Ciudad Universitaria Meléndez, Calle 13 No 100-00, Cali-Colombia. \\ (e-mail: guillermo.aponte@correounivalle.edu.co). \\ (2) Universidad Carlos III, Av. Universidad 30-28911 Leganes, Madrid-España.
}

Recibido Jun. 16, 2010; Aceptado Ago. 06, 2010; Versión Final recibida Mar. 18, 2011

\begin{abstract}
Resumen
Este artículo presenta la implementación de un modelo para el análisis del comportamiento en frecuencia de los parámetros de un transformador eléctrico. El modelo se obtiene a partir de la curva de la prueba de Respuesta en Frecuencia del transformador (FRA). Esta prueba consiste básicamente en obtener una característica del transformador y comparar el resultado con una referencia del mismo, tomada anteriormente y considerada como normal. Las diferencias pueden indicar algún tipo de daño en el transformador. Se hace la descripción del modelo, de la manera como se realizó su implementación, del procedimiento para obtener sus parámetros y de su aplicación en el análisis de variaciones en las curvas obtenidas en los ensayos de FRA. Este modelo es una herramienta que puede emplearse como ayuda para identificar problemas de tipo mecánico en el transformador.
\end{abstract}

\section{Implementation of an Electric Transformer Model for its Frequency Response Analysis}

\begin{abstract}
This article presents the implementation of a model for the analysis of the frequency behavior of a transformer. The model is obtained from the Frequency Response Test (FRA). This test consists basically of obtaining the characteristics of the transformer and comparing the results with those of a reference transformer, which are previously known and considered as normal- The differences indicate that the transformer has certain damage. The article describes the model, its implementation, the procedure to obtain its parameters and its application in the analysis of the curve variations obtained by the FRA tests. This model represents a good tool that can be used as support to identify some type of mechanical problem in the transformer.
\end{abstract}

Keywords: transformer, transformer parameters, frequency response, FRA 


\section{INTRODUCCION Y DESCRIPCION DEL FRA}

El transformador es uno de los equipos más importantes en los sistemas eléctricos tanto por su alto costo individual, como por ser pieza clave para brindar un adecuado servicio. Mantener el transformador en buenas condiciones, es fundamental para hacer un eficiente aprovechamiento del equipo durante toda su vida útil. La respuesta de frecuencia, técnica conocida como FRA (por sus siglas en inglés, Frequency Response Analysis), es una prueba, para evaluar la condición mecánica de los transformadores, que consiste básicamente en obtener un registro en frecuencia del transformador y comparar el resultado con una referencia del mismo, tomada anteriormente y considerada como normal. Las diferencias pueden indicar algún tipo de daño en el transformador. La respuesta en frecuencia, puede obtenerse por el método de Impulso o por el de Barrido, este último denominado como SFRA (Sweep Frequency Response Analysis). En el SFRA se obtiene la impedancia o la función de transferencia del transformador, mediante la excitación con una señal periódica senoidal en un amplio rango de frecuencias. La Amplitud y fase de señales obtenidas de los terminales seleccionados de los transformadores se representan directamente en función de la frecuencia (Dick y Erven, 1978).

La respuesta en frecuencia es una "huella digital" del transformador, ya que es única. Cualquier variación en el registro, indicará un cambio mecánico o eléctrico en su interior. La prueba se repite en el tiempo y dos o más huellas se comparan para hacer un diagnostico, como se muestra en la Fig. 1 (Ryder, 2003).

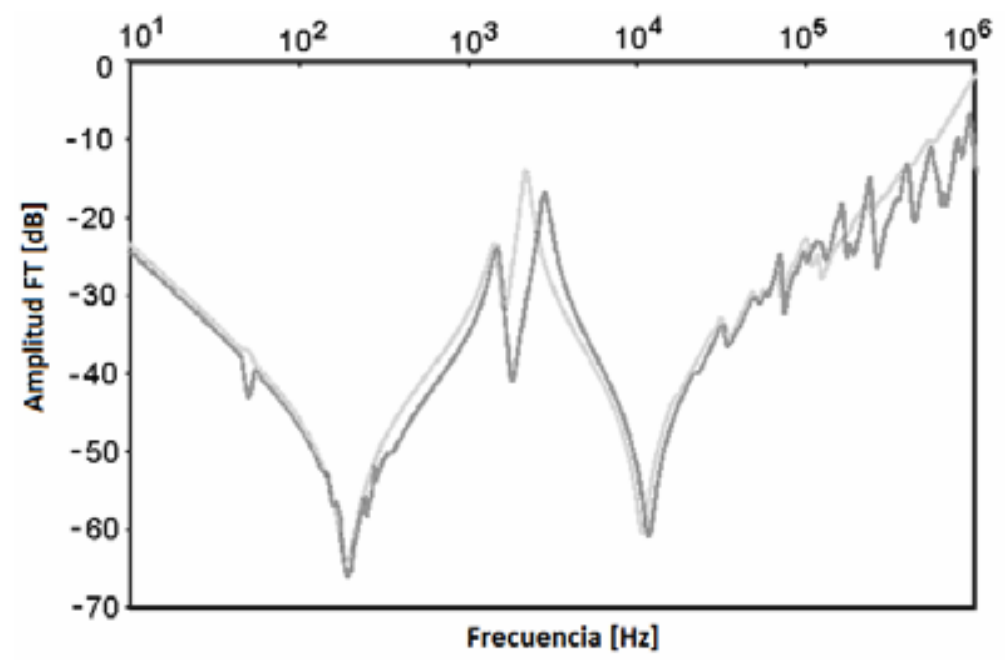

Fig. 1. Comparación de curvas de respuesta en frecuencia.

En la actualidad no existe una norma aprobada internacionalmente para la ejecución de la prueba FRA, por lo que se toman como referencia documentos técnicos propuesto por organismos internacionales (CIGRE, 2008), en discusión (IEEE, 2009; IEC, 2009) y normas nacionales (DL/T, 2004).

En el mercado existen varios equipos que permiten obtener el registro de FRA de los transformadores. Si bien es aceptado que una variación en la respuesta es indicativo de un cambio interno y por lo tanto de una posible causa de falla, es difícil interpretar cuantitativa y cualitativamente de manera precisa los resultados del FRA, ya que no existen reglas o guías que definan exactamente a qué corresponde una determinada variación; es este el aspecto critico de esta técnica, pues a partir del análisis de las variaciones se debe decidir si el transformador está en condiciones de seguir trabajando o si debe sacarse de servicio. Para realizar la comparación, adicionalmente al análisis visual, se han propuesto métodos que emplean coeficientes de correlación (Ryder, 2003), la desviación espectral (DL/T, 2004), y la utilización de modelos equivalentes (Pleite, 2002; Pong, 2004; Bjerkan et al., 2005; Herszterg et al., 2005; Rahimpour et al., 2003; Florkowski y Furgal, 2009). 
Una alternativa para la obtención de relaciones causa efecto entre fallas y respuesta, es la de emplear modelos del transformador donde se simulen cambios y se identifiquen sus efectos en el FRA. Esta metodología es aplicable de manera general, ya que el análisis se hace en el modelo propio de cada transformador, debiendo el modelo permitir realizar una asociación de sus parámetros con los componentes físicos del transformador. Un modelo se puede obtener a partir de datos constructivos, para lo cual se requiere tener una muy detallada y completa información del transformador, lo que implica trabajar en asocio con el fabricante, quien tiene todos los datos de su diseño y construcción. También se han planteado alternativas para elaborar modelos a partir de curvas obtenidas en pruebas de campo (Pleite, 2002; Pong, 2004), lo que facilita su aplicación para transformadores instalados, en los cuales es difícil disponer de la información sobre sus parámetros constructivos.

El presente trabajo muestra la implementación de un modelo que se compone de celdas RLC, las cuales representan un determinado ancho de banda de la respuesta en frecuencia, caracterizado por un punto de resonancia; el orden del modelo depende de la cantidad de frecuencias de resonancia identificadas. Las variaciones de los parámetros del modelo, correspondiente a dos curvas de FRA son utilizadas para realizar un diagnostico de la condición del transformador; por lo que el modelo puede ser muy útil para el análisis de las curvas FRA de transformadores.

\section{DESCRIPCION DEL MODELO RLC}

El modelo implementado (Pleite, 2000), se construye a partir de una respuesta en frecuencia medida en el transformador y consiste básicamente en una serie de celdas conformadas por tres parámetros eléctricos, $R, L, C$, que permite reproducir la curva de respuesta en frecuencia obtenida en una prueba. Estos parámetros se encuentran conectados en paralelo en cada celda, como se muestra en la Fig. 2, para un modelo de 4 celdas. Este circuito modela la respuesta en frecuencia producida por una fase del transformador.

Las celdas están formadas por tres elementos pasivos básicos, la inductancia que representa el almacenamiento de campo magnético, la capacitancia que representa el almacenamiento de campo eléctrico y la resistencia que representa las pérdidas de potencia. La obtención de los parámetros eléctricos, R, L, C de cada celda se hace a partir de una respuesta en frecuencia medida, como la mostrada en la Fig. 3, en la cual existen diversos picos y valles a lo largo de todo el ancho de banda de frecuencia.

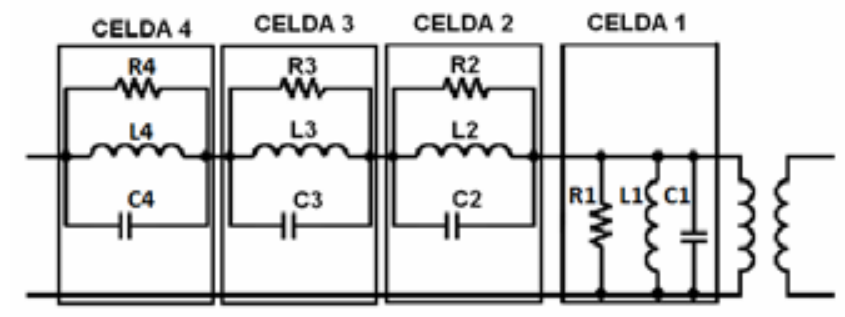

Fig. 2. Modelo de celdas RLC

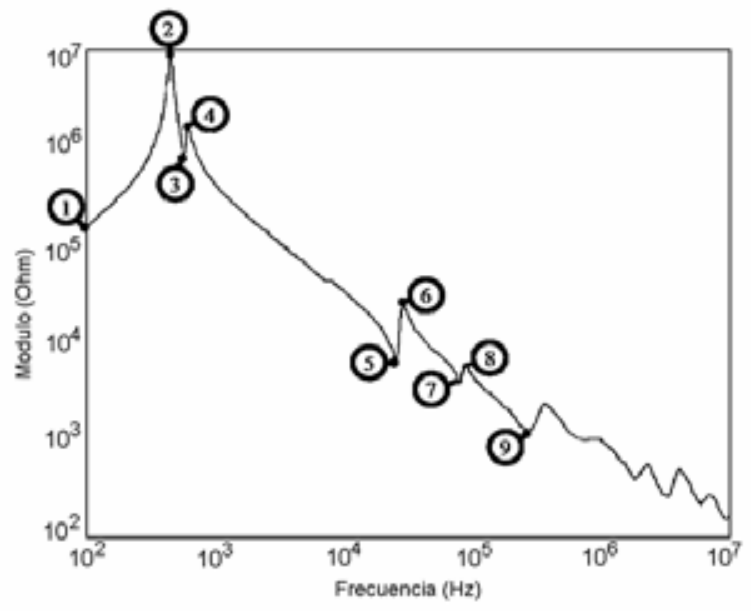

Fig. 3. Respuesta en frecuencia medida

El primer valle pico valle (1-2-3) está representado por la primera celda del modelo, lo mismo ocurre para el resto de celdas del modelo (3-4-5), (5-6-7) ... 
La respuesta en frecuencia del modelo, tendrá también diversos picos y valles a lo largo de todo el ancho de banda (Fig 4). El ancho de banda A1-B1 está asociado básicamente a la respuesta de la primera celda, donde L1 corresponde al flanco de subida, C1 al flanco de bajada y R1 al punto de resonancia. Esto se repite para el resto de celdas del modelo. En cada banda de frecuencia del modelo es reconocida una celda, siendo el resto transparentes (se comportan como un corto circuito).

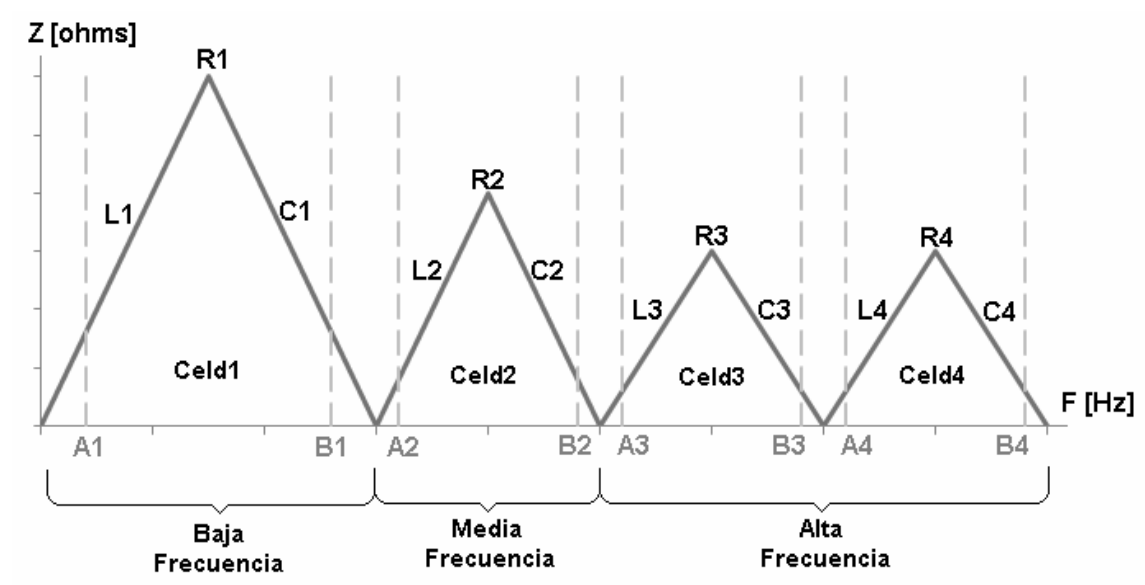

Fig 4. Respuesta de un modelo de celdas

Cada celda se asocia principalmente con un aspecto del comportamiento del transformador: la que está en la banda de baja frecuencia (C1) con efectos en el núcleo, la que está en bandas intermedias de frecuencia (C2) con efectos principales en el devanado y las que están en la banda de alta frecuencia (C3 y C4) con efectos menores en el devanado.

Para elaborar el modelo se requiere contar con una respuesta en frecuencia medida en el transformador, como la mostrada en la figura 1, pero sus datos deben ser entregados en forma de una tabla de impedancia $(\mathrm{R}+\mathrm{jX})$ y frecuencia $(\mathrm{Hz})$. Cada celda se representa con su admitancia equivalente Ec. 1.

$Y\left(\omega, R_{k}, C_{k}, L_{k}\right)=\frac{1}{R_{k}}+j \omega C_{k}+\frac{1}{j \omega L_{k}}(k=1,2 \ldots)$

Donde k representa el número de la celda.

El error entre la respuesta real $Y_{R}$ y la respuesta del circuito modelado $Y_{M}$ en cada punto es:

$E\left(R_{s}, C_{x} L\right)=\sum_{i=1}^{m} \mid Y_{R_{i}}\left(\omega_{i}\right)-Y_{M}\left(\omega_{i}, R_{s} C_{x} L X^{2}\right.$

$Y_{R}\left(\omega_{R}\right)=G_{R}\left(\omega_{i}\right)+j B_{R}\left(\omega_{i}\right)$

Donde $Y_{B}\left(\omega_{i}\right)$ es la respuesta de admitancia medida, designada como real en coordenadas cartesianas; $G_{R}\left(\omega_{i}\right)$ y $B_{R}\left(\omega_{i}\right)$ son la conductancia y susceptancia medidas en cada punto.

Los parámetros del modelo se obtienen a partir de los datos de impedancia y frecuencia medidos, Ec. 5, Ec 6, y Ec 7, estas ecuaciones se obtienen de la minimización de la función de error, $E(R, C, E)$, por medio del sistema de ecuaciones resultado de:

$\nabla E\left(R_{r}, C, E\right)=0 \rightarrow \nabla^{2} E\left(R_{r}, C, L\right)>0$

El desarrollo matemático del modelo, se explica con más detalle en (Pleite, 2000). 


$$
\begin{aligned}
& R=\frac{m}{\sum_{i=1}^{m} G_{R}\left(\omega_{i}\right)} \\
& L=\frac{\sum_{i=1}^{m} \omega_{i}^{2} \cdot \sum_{i=1}^{m} \frac{1}{\omega_{i}^{2}}-m^{2}}{m \cdot \sum_{i=1}^{m} \omega_{i} B_{R}\left(\omega_{i}\right)-\sum_{i=1}^{m} \frac{B_{R}\left(\omega_{i}\right)}{\omega_{i}} \cdot \sum_{i=1}^{m} \omega_{i}^{2}} \\
& C=\frac{\sum_{i=1}^{m} \omega_{i} E_{R}\left(\omega_{i}\right) \cdot \sum_{i=1}^{m} \frac{1}{\omega_{i}^{2}}-m \cdot \sum_{i=1}^{m} \frac{B_{R}\left(\omega_{i}\right)}{\omega_{i}}}{\sum_{i=1}^{m} \omega_{i}^{2} \cdot \sum_{i=1}^{m} \frac{1}{\omega_{i}^{2}}-m^{2}}
\end{aligned}
$$

Donde $m$ es el número de puntos incluidos en el ancho de banda asignada.

Ya que las celdas no se comportan completamente como cortocircuitos en frecuencias fuera de su banda, se emplea un procedimiento iterativo, para aproximar el modelo a la curva real:

i) Inicialmente se calculan los parámetros de la celda de mayor frecuencia, en la banda de frecuencias asignada.

ii) El valor de los parámetros del resto de las celdas, se ajustan secuencialmente de acuerdo a su ancho de banda asignado, descontando el efecto de las celdas de más alto orden.

iii) En las iteraciones siguientes, los parámetros de cada celda son recalculados, en su ancho de banda asignado, descontando el efecto del resto de las celdas

\section{IMPLEMENTACIÓN DEL MODELO}

El algoritmo para obtener el modelo, inicia convirtiendo los datos de FRA a impedancia, luego se seleccionan los anchos de banda de cada celda y por último, se realiza un procedimiento iterativo para aproximar el modelo a la curva real.

\section{Conversión a Impedancia}

Por lo general los equipos comerciales obtienen la curva FRA a través de la función de transferencia, ya que este es el método más sensible para detectar fallas en el transformador (Jayasinghe et al., 2006). Para obtener el modelo es necesario convertir los datos de la función de transferencia a impedancia. El circuito de medición básica se muestra en la figura 5 , donde $\mathrm{S}$ es la señal inyectada, $R$ es la señal de referencia, T la señal medida, Zs es la impedancia de entrada del equipo analizador y ZT es la impedancia del devanado bajo ensayo.

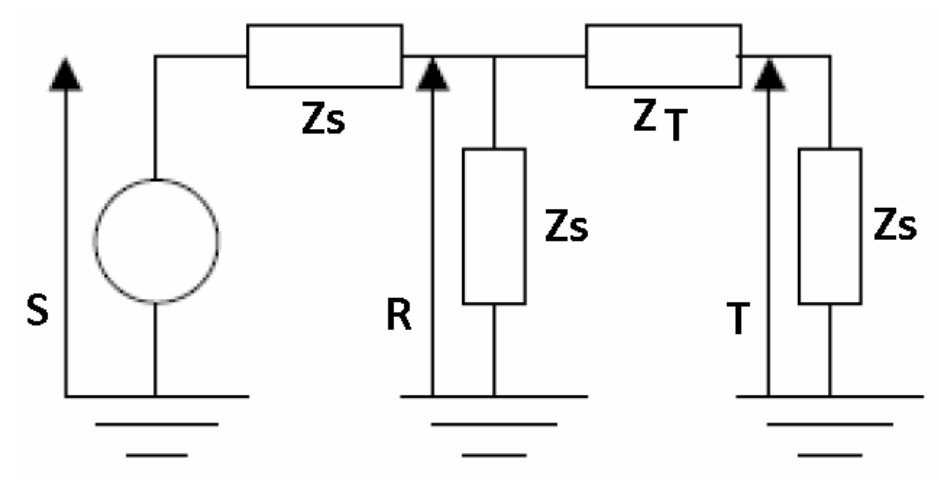

Fig. 5. Circuito de medición básica 
La respuesta en dB es calculada a través de la siguiente ecuación:

$$
\begin{aligned}
& E T_{i}[d B]=20 \cdot \log _{10}\left(\frac{V_{\text {ouE }}}{V_{\text {in }}}\right) \\
& \frac{V_{\text {ouE }}}{V_{i n}}=\exp \left(\frac{\text { in } 10}{20} \cdot F T_{i}[d B]\right)
\end{aligned}
$$

La Impedancia de entrada Zs del equipo analizador es por lo general de 50 ohm. De esta medida se obtiene:

$$
\left(\frac{V_{\text {out }}}{V_{\text {in }}}\right) \angle \theta=\frac{50}{50+Z \angle \varphi}
$$

Dónde $F T$ es la función de transferencia y $z$ es la función de impedancia dependiente de la

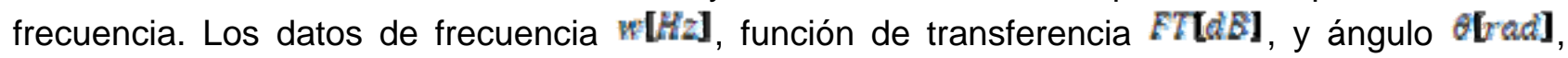
obtenidos a través de la medición FRA, son convertidos a impedancia a través de la siguiente ecuación:

$Z_{\angle \varphi}=\frac{50}{\left(\frac{V_{\text {owt }}}{V_{t n}}\right) \angle \theta}-50$

\section{Selección de anchos de banda}

A cada celda se le asigna un ancho de banda, el cual debe contener un pico de resonancia. En la Fig. 6 se muestra un caso de 4 celdas, en donde se muestran los anchos de banda asignados a cada celda.

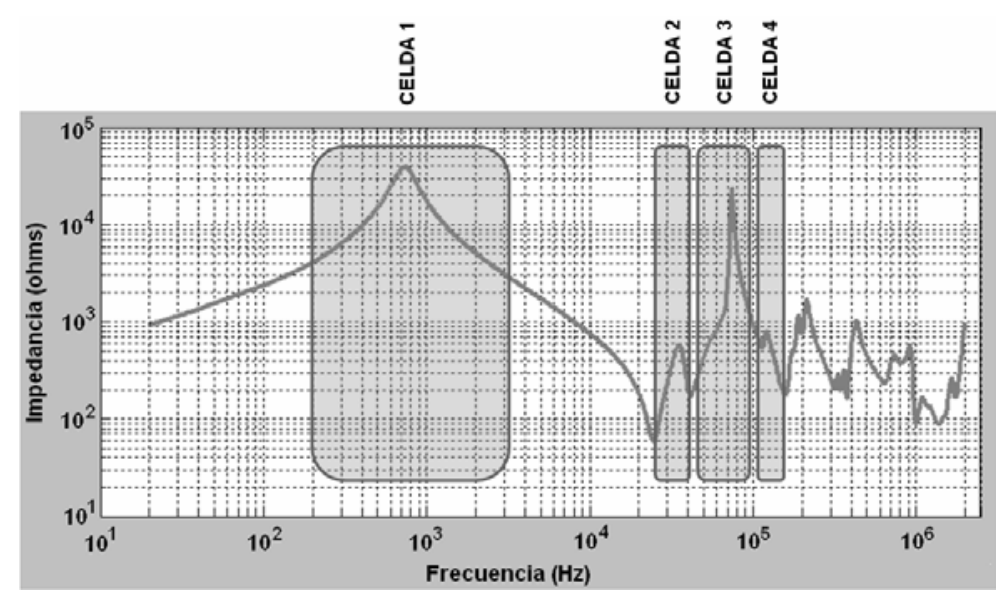

Fig. 6. Selección de anchos de Banda

La posición en la matriz, del punto inicial y el punto final de los anchos de banda escogidos se guardan en un vector de posiciones. Luego se relaciona la matriz de datos, con el vector de posiciones y se calcula el número de puntos de cada ancho de banda, como se muestra en la Tabla1.

\section{Procedimiento Iterativo}

El procedimiento iterativo consta de 4 pasos principales y comienza en la celda de mayor orden $\mathrm{C} 4$, siendo inicialmente Z1, Z2, Z3 y Z4 iguales a cero. En cada celda se calcula una impedancia de referencia Zref, la cual se utiliza para obtener los parámetros RLC. 
Tabla 1. Matriz de datos

\begin{tabular}{ccccc}
\hline Celda & $\begin{array}{c}\text { Frecuencia } \\
{[\mathrm{Hz}]}\end{array}$ & $\mathrm{R}[\Omega]$ & $\mathrm{X}[\Omega]$ & $\begin{array}{c}\text { Numero } \\
\text { de puntos }\end{array}$ \\
\hline \multirow{3}{*}{ Celda1 } & $\mathrm{F}(\mathrm{A} 1)$ & $\mathrm{R}(\mathrm{A} 1)$ & $\mathrm{X}(\mathrm{A} 1)$ & \\
& $\vdots$ & $\vdots$ & $\vdots$ & $\mathrm{m} 1$ \\
& $\mathrm{~F}(\mathrm{~B} 1)$ & $\mathrm{R}(\mathrm{B} 1)$ & $\mathrm{X}(\mathrm{B} 1)$ & \\
Celda2 & $\mathrm{F}(\mathrm{A} 2)$ & $\mathrm{R}(\mathrm{A} 2)$ & $\mathrm{X}(\mathrm{A} 2)$ & \\
& $\vdots$ & $\vdots$ & $\vdots$ & $\mathrm{m} 2$ \\
& $\mathrm{~F}(\mathrm{~B} 2)$ & $\mathrm{R}(\mathrm{B} 2)$ & $\mathrm{X}(\mathrm{B} 2)$ & \\
Celda3 & $\mathrm{F}(\mathrm{A} 3)$ & $\mathrm{R}(\mathrm{A} 3)$ & $\mathrm{X}(\mathrm{A} 3)$ & \\
& $\vdots$ & $\vdots$ & $\vdots$ & $\mathrm{m} 3$ \\
& $\mathrm{~F}(\mathrm{~B} 3)$ & $\mathrm{R}(\mathrm{B} 3)$ & $\mathrm{X}(\mathrm{B} 3)$ & \\
Celda 4 & $\mathrm{F}(\mathrm{A} 4)$ & $\mathrm{R}(\mathrm{A} 4)$ & $\mathrm{X}(\mathrm{A} 4)$ & \\
& $\vdots$ & $\vdots$ & $\vdots$ & $\mathrm{m} 4$ \\
& $\mathrm{~F}(\mathrm{~B} 4)$ & $\mathrm{R}(\mathrm{B} 4)$ & $\mathrm{X}(\mathrm{B} 4)$ & \\
\hline
\end{tabular}

Paso1: Cálculo de las impedancias de cada celda

Cálculo de los parámetros de la Celda 4:

Se calcula la impedancia Zref4 en el ancho de banda A4-B4, como la impedancia Zreal de la curva descontando el efecto de las otras celdas y excluyendo la impedancia de la celda bajo análisis, por lo tanto:

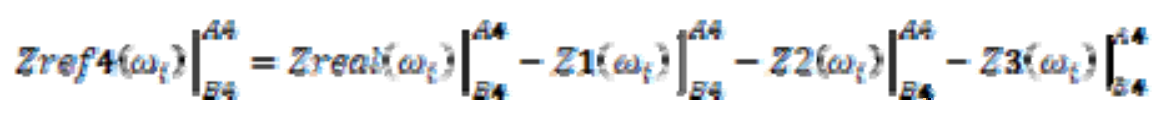

La admitancia de referencia en el ancho de banda A4-B4 es igual a:

$\left.\operatorname{Yref} 4\right|_{B 4} ^{A 4}=\left.G\left(\omega_{i}\right)\right|_{B 4} ^{A 4}+\left.j B\left(\omega_{i}\right)\right|_{B 4} ^{A 4}=\left.\frac{1}{Z r e f 4}\right|_{\bar{E} 4} ^{A 4}$

A través de la admitancia de referencia se obtienen los parámetros R4, C4 y L4, empleando las ecuaciones 5, 6 y 7, teniendo en cuenta el número de puntos $\mathrm{m} 4$ de este rango. Se calcula la impedancia total de la celda Z4, como la suma en paralelo de R4, C4 y L4 para el ancho de banda determinado para la celda4, por lo tanto:

$\left.Z 4\right|_{B 4} ^{A 4}=\frac{1}{\left(\frac{1}{R^{4}}\right)+j \omega C 4+\frac{1}{j \omega L 4}}$

Los mismos pasos se realizan para las celdas 3,2 y 1 .

Paso 2. Cálculo del error relativo promedio ERP

Se calcula la función de impedancia del modelo, en todo en rango de frecuencia de la curva, como:

$\left.Z m\right|_{B 4} ^{A 1}=Z 1+Z 2+Z 3+Z 4$

El error ERP es igual a:

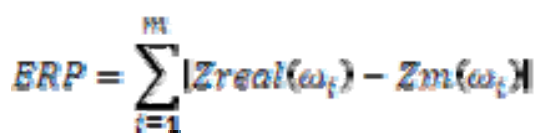


Donde Zreal es la impedancia medida y $\mathrm{Zm}$ es la impedancia modelada.

Paso 4. Evaluación del error:

Se evalúa el Error ERP, cometido por la respuesta del modelo respecto a la respuesta medida. Si el error no es suficientemente bajo, los parámetros del modelo son recalculados retornando de nuevo al paso1. En la Fig. 7 se muestra en un diagrama de bloques del algoritmo.

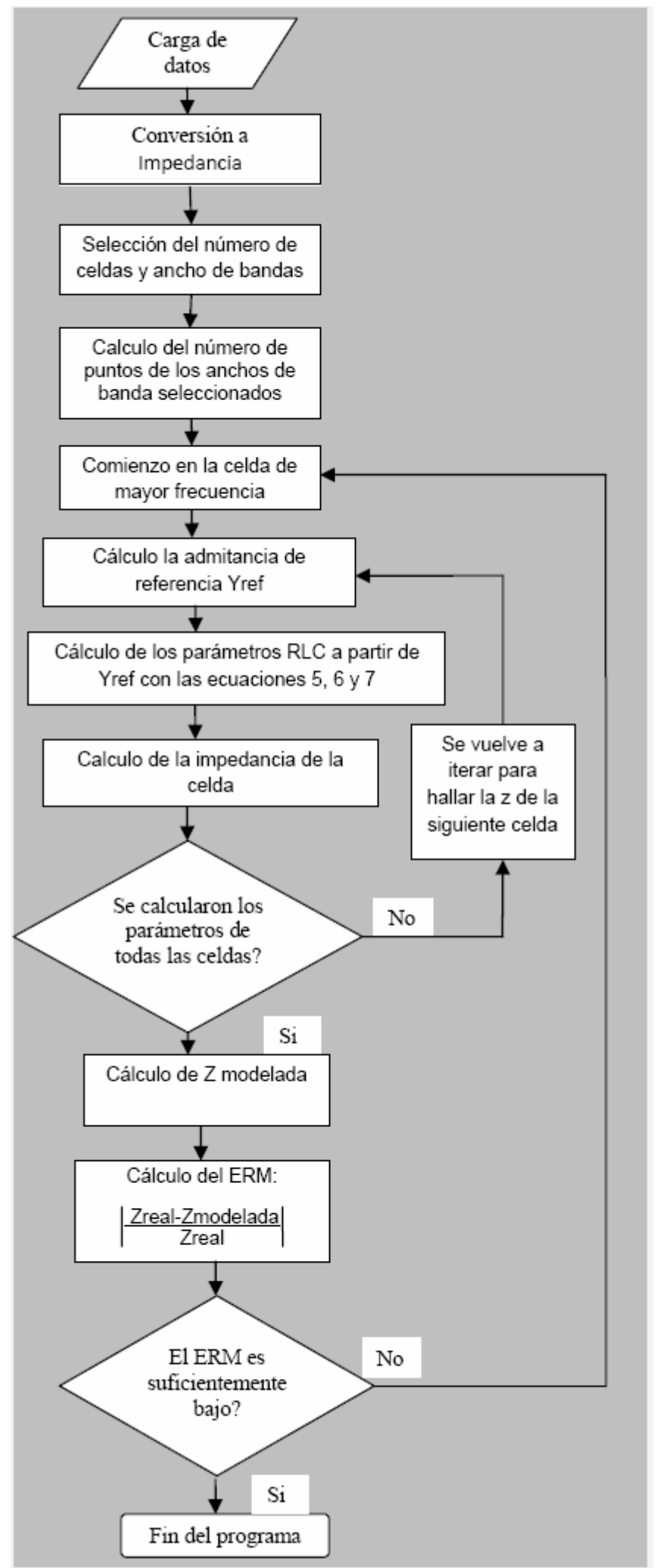

Fig. 7. Diagrama del algoritmo 


\section{VALIDACIÓN DEL MODELO}

El algoritmo se implementó para obtener el modelo de celdas de un transformador de 12 MVA, cuyas curvas de respuesta en frecuencia medida y modelada, se muestran en la Fig. 8.

Se escogieron 4 celdas para modelar los primeros 4 picos de la curva. El modelado de la curva presentó un error relativo promedio de $10 \%$, observándose su mayor discrepancia en la parte inicial, lo que indica que no se ajusta de manera correcta en esta zona.

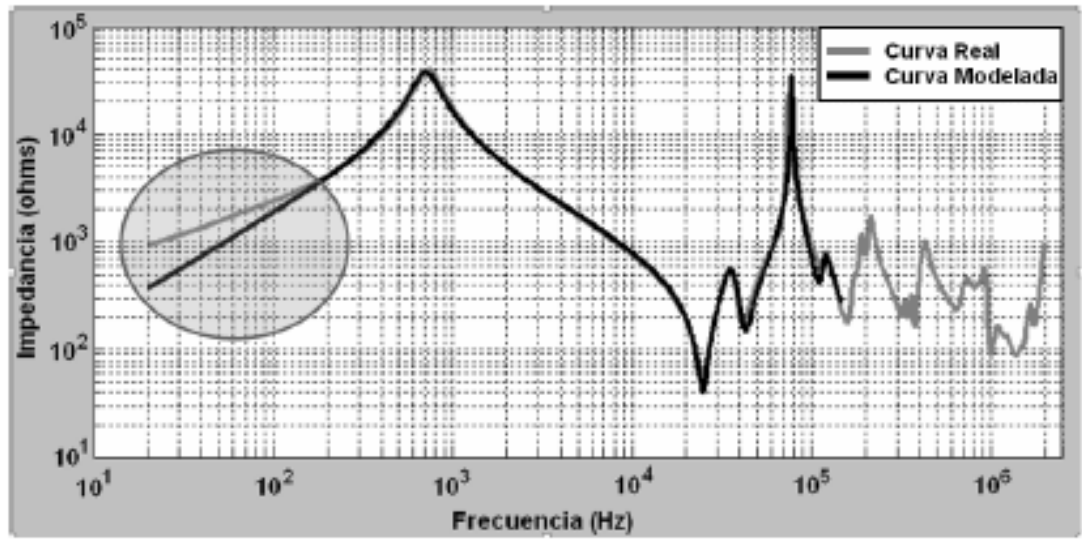

Fig. 8. Curvas de FRA medida y modelada.

En la figura 9 se observa la curva de fase para el primer pico de resonancia, donde se puede observar que la curva del modelo inicia en 90 grados, lo que representa un comportamiento totalmente inductivo a bajas frecuencias. Sin embargo la curva medida, tiene un inicio de fase menor de 90 grados, lo que muestra un pequeño efecto resistivo, que produce la diferencia de fase inicial con el modelo.

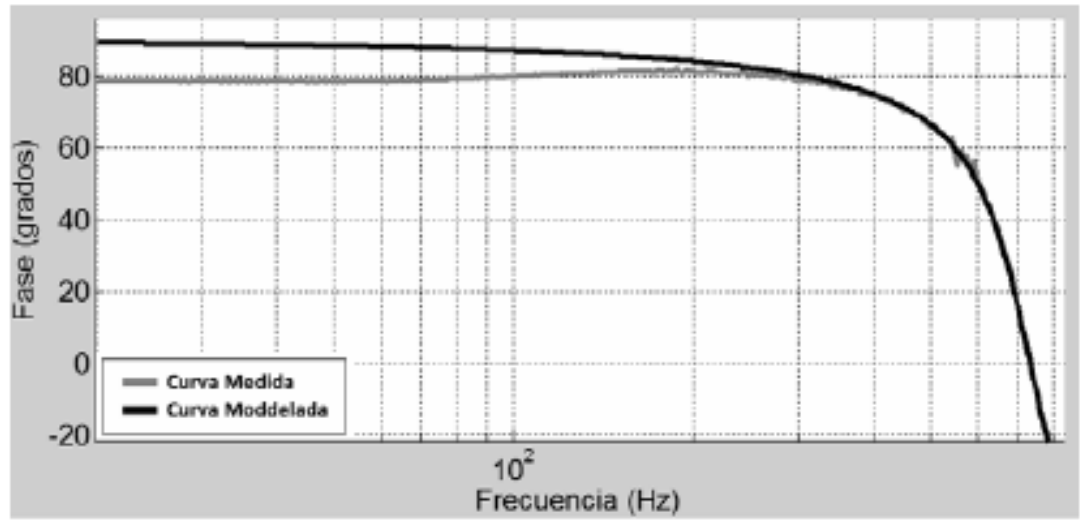

Fig. 9. Curvas de Fase de la respuesta medida y modelado.

La diferencia se debe a que el modelo de celdas no incluye la resistencia en corriente continua de los devanados, esto se puede observar en el circuito equivalente en corriente continua del modelo (Fig. 10), el cual es simplemente un cortocircuito, debido a que las reactancias inductivas son cero.
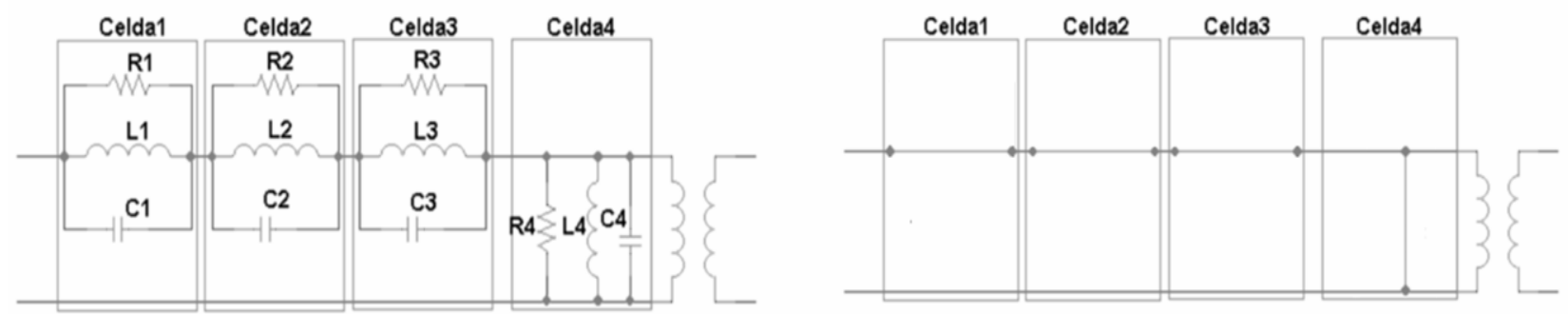

Fig. 10. Modelo de 4 celdas y su comportamiento en corriente continúa 
La zona inicial del primer pico del registro FRA, está influenciado por el efecto de la resistencia en continua de los arrollamientos, sin embargo a medida que se aumenta la frecuencia, empieza a predominar la resistencia de pérdidas del núcleo. Por lo general, es muy pequeño el efecto de la resistencia Rdc, sobre los parámetros de la primera celda obtenidos en el modelado, debido a que su valor es bajo.

\section{APLICACIÓN DEL MODELO PARA DIAGNÓSTICO}

La metodología de diagnostico a través del modelo requiere de dos registros, uno de la curva de respuesta en frecuencia del transformador en estado sano y otro del transformador en estado de falla. Cada curva de FRA se modela por separado usando el algoritmo previamente descrito, para encontrar los parámetros de todas las celdas del modelo.

Por lo tanto se tendrá un modelo del transformador en "estado sano" y otro en "estado de falla" Fig. 11.

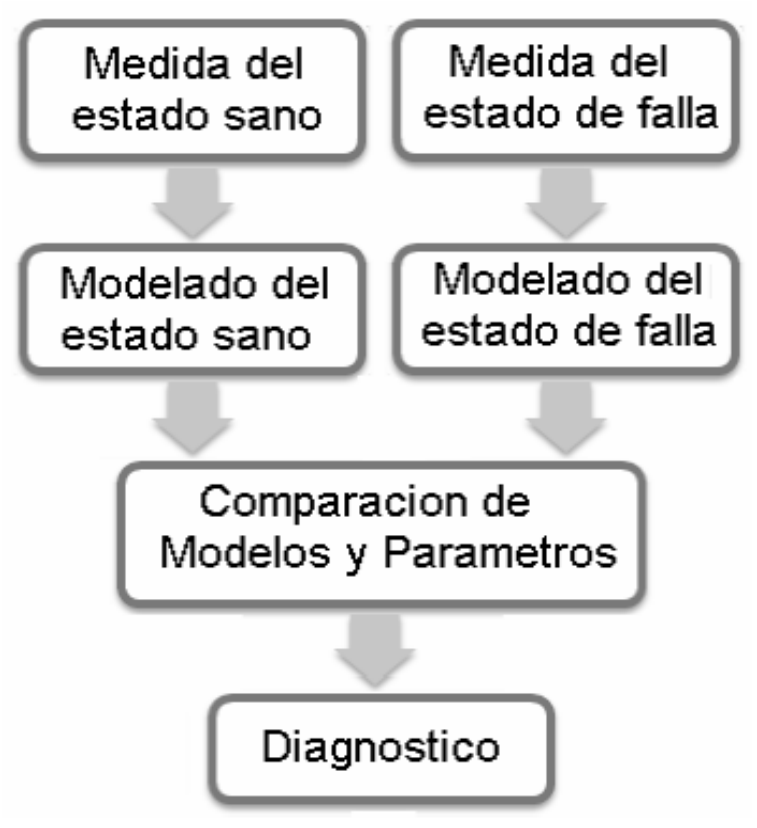

Fig. 11. Aplicación del modelo

Finalmente, los parámetros de los modelos son comparados y se calcula su diferencia en porcentaje. Las diferencias en los parámetros de las celdas pueden indicar algún tipo de daño en el transformador.

Caso de estudio:

A continuación se presenta la aplicación del modelo en un transformador de $880 \mathrm{KVA}, 13.86 \mathrm{kV} /$ $500 \mathrm{~V}$, YnD11, con núcleo tipo triangular. A este transformador se le realizó la prueba FRA inicial, obteniéndose la curva en estado sano para la fase $\mathrm{X} 1-\mathrm{X} 0$, a partir de la cual se estimaron los valores de las celdas (modelo-sano).

Posteriormente se realizó un desplazamiento axial del devanado de baja en la fase X1-X0, levantándolo $10 \mathrm{~cm}$, con respecto al devanado de alta, obteniéndose una nueva curva de FRA (modelo-falla). Las curvas en estado sano y estado de falla se muestran en la Fig. 12. Para modelar las curvas en estado sano y de falla, se escogieron 5 celdas, con los anchos de banda mostrados en la figura 13. En la figura 14 se muestran las curvas medida y modelada, para la fase $\mathrm{X} 1-\mathrm{X} 0$, en estado normal 


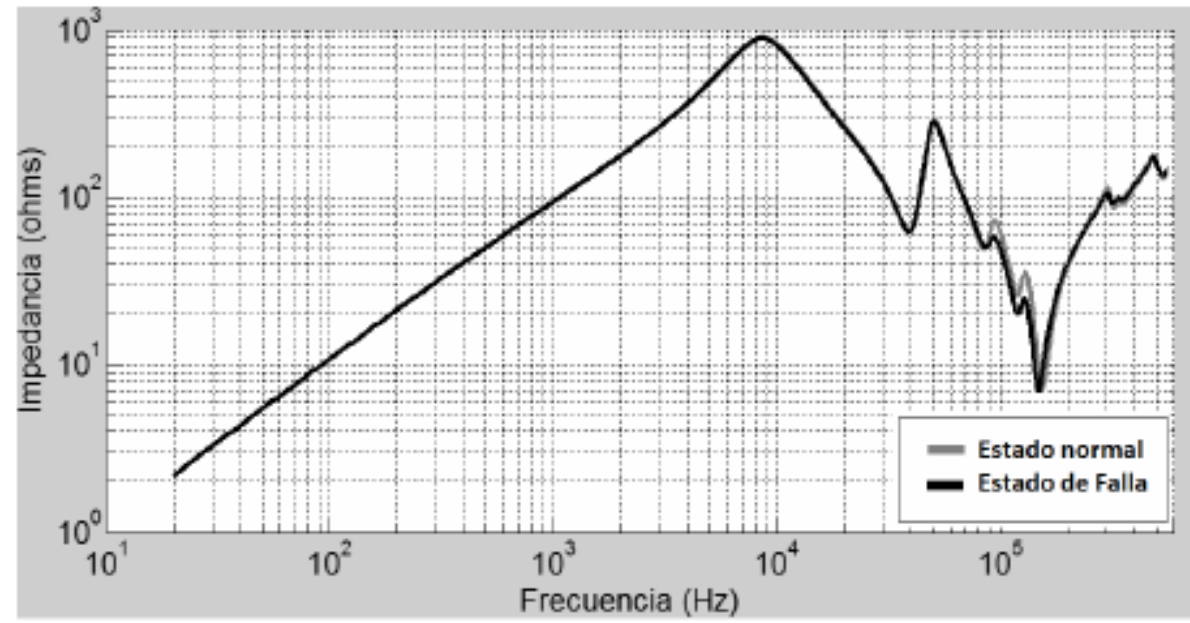

Fig. 12. Curvas FRA medidas en estado sano y en estado de Falla, para la fase X1-X0.

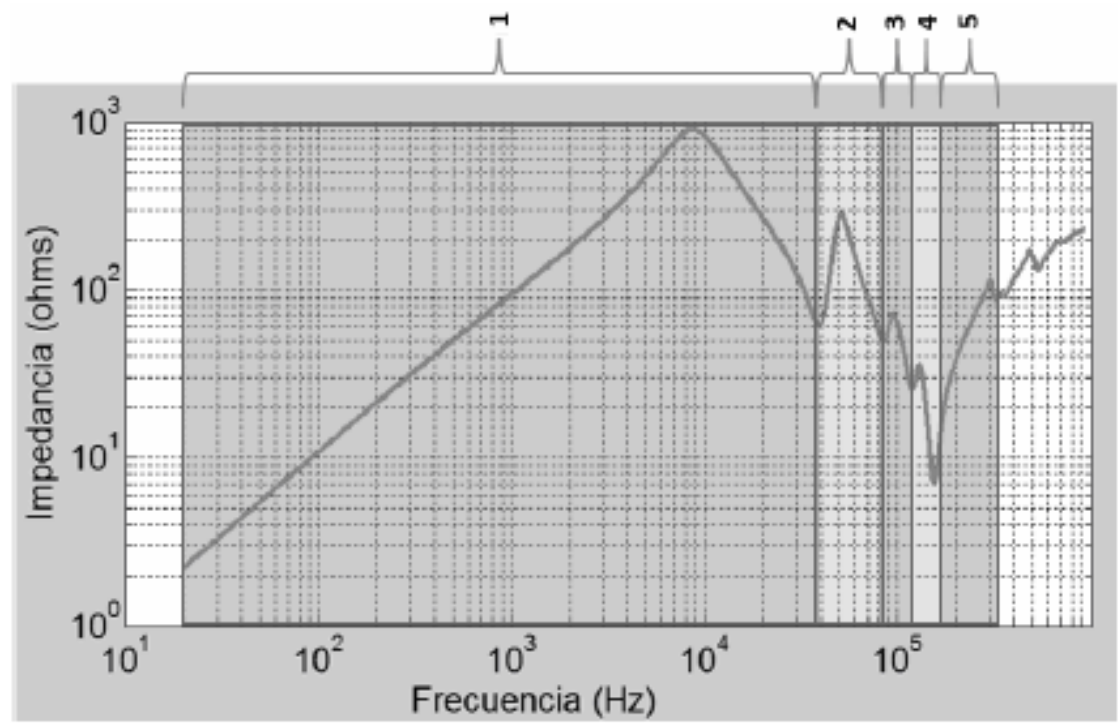

Fig. 13. Selección de los anchos de banda para el modelado.

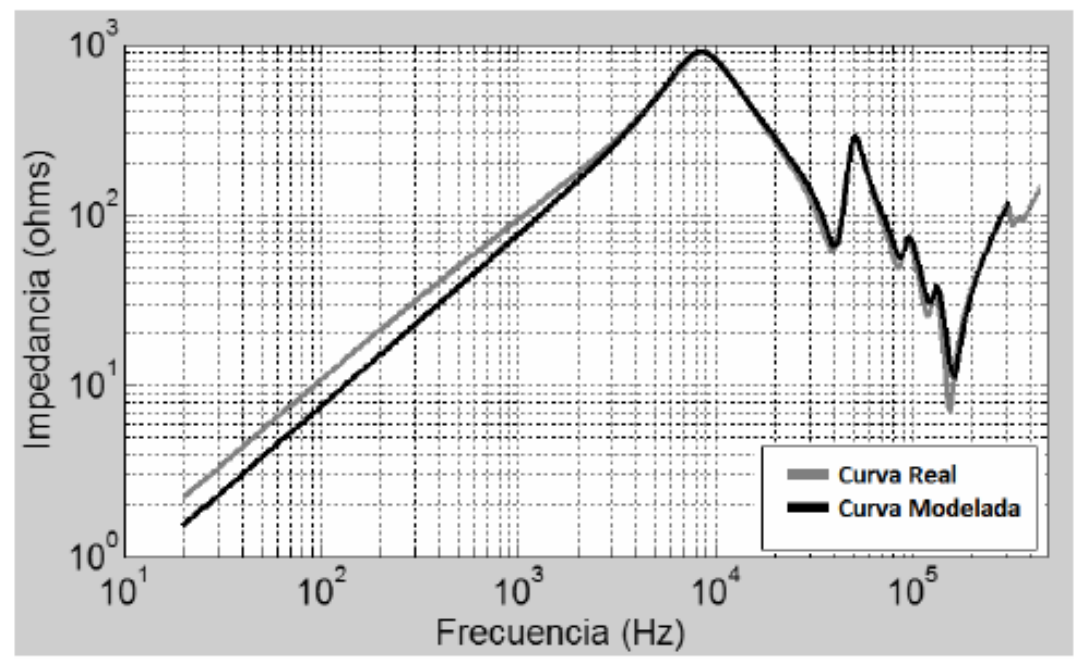

Fig. 14. Curvas medida y modelada para la fase X1-X0 en estado normal. 
Inicialmente se realizó un análisis de las curvas a través de dos índices estadísticos; la desviación espectral y el coeficiente de correlación. Las bandas de frecuencias seleccionadas para aplicar los índices, fueron seleccionadas según se sugiere en (Ryder, 2003). En la tabla 2, se muestran los valores de estos índices estadísticos aplicados a las curvas, en estado sano y en estado de falla. Para este caso los índices estadísticos no muestran una variación considerable, por lo que no pueden detectar la falla.

Tabla 2: Valores de los índices estadísticos aplicados

\begin{tabular}{|c|c|c|}
\hline $\begin{array}{c}\text { Banda de } \\
\text { Frecuencia }\end{array}$ & $\begin{array}{c}\text { Coeficiente de } \\
\text { Correlación }\end{array}$ & $\begin{array}{c}\text { Desviación } \\
\text { Espectral }\end{array}$ \\
\hline $20 \mathrm{~Hz}-100 \mathrm{~Hz}$ & 0.999 & 0.004895 \\
\hline $100 \mathrm{~Hz}-1 \mathrm{kHz}$ & 1.000 & 0.000413 \\
\hline $1 \mathrm{kHz}-10 \mathrm{kHz}$ & 1.000 & 0.001687 \\
\hline $10 \mathrm{kHz}-100 \mathrm{kHz}$ & 0.999 & 0.020931 \\
\hline $100 \mathrm{kHz}-1 \mathrm{MHz}$ & 0.998 & 0.046130 \\
\hline
\end{tabular}

En la Tabla 3 se muestran los parámetros de las celdas obtenidos con el modelo para los dos casos, junto con la diferencia porcentual de los mismos. Las celdas 1 y 2 no muestran una variación considerable, lo que indica que el sistema magnético del transformador se encuentra en buen estado, además no se presenta deformaciones mayores en el devanado; sin embargo se observan grandes variaciones en las celdas 3,4 y 5, que indica una deformación menor en la fase X1-X0.

Tabla 3: Valores de los parámetros del modelo

\begin{tabular}{||c|c|c|c||}
\hline & Estado sano & Estado de Falla & Diferencia \% \\
\hline \hline L1 & 0.01185 & 0.01189 & 0.338 \\
\hline R1 & 900.255 & 894.887 & -0.596 \\
\hline C1 & $2.918 \mathrm{E}-08$ & $2.984 \mathrm{E}-08$ & 2.262 \\
\hline \hline L2 & 0.0001383 & 0.0001366 & -1.229 \\
\hline R2 & 252.275 & 247.001 & -2.091 \\
\hline C2 & $7.321 \mathrm{E}-08$ & $7.529 \mathrm{E}-08$ & 2.841 \\
\hline \hline L3 & $1.090 \mathrm{E}-05$ & $6.721 \mathrm{E}-06$ & -38.339 \\
\hline R3 & 38.859 & 22.483 & -42.142 \\
\hline C3 & $2.633 \mathrm{E}-07$ & $4.287 \mathrm{E}-07$ & 62.818 \\
\hline \hline L4 & $2.8582 \mathrm{E}-06$ & $2.11 \mathrm{E}-06$ & -26.177 \\
\hline R4 & 19.8965 & 13.369 & -32.807 \\
\hline C4 & $5.266 \mathrm{E}-07$ & $7.105 \mathrm{E}-07$ & 34.922 \\
\hline \hline L5 & $5.922 \mathrm{E}-05$ & $6.119 \mathrm{E}-05$ & 3.327 \\
\hline R5 & 586.728 & 735.3937 & 25.338 \\
\hline C5 & $1.067 \mathrm{E}-09$ & $6.442 \mathrm{E}-10$ & -39.625 \\
\hline \hline
\end{tabular}




\section{CONCLUSIONES}

La metodología propuesta para la implementación del modelo permite obtener los parámetros de cada celda de manera que se pueden reproducir los picos de resonancia de la curva SFRA con un error aceptable. El modelo ayuda en la interpretación física de los cambios en los parámetros del transformador asociados a la variación de su respuesta en frecuencia, para realizar un análisis cualitativo; además el modelo resalta las pequeñas diferencias existentes en dos registros, permitiendo también un análisis cuantitativo.

Por otro lado, el modelo de celdas muestra ser sensible a los cambios en la curva de SFRA, por lo que puede ser útil al evaluar el alcance de una falla; sin embargo se requiere de más investigación, para obtener reglas que asocien las variaciones en los parámetros de las celdas con los cambios físicos en el transformador. Además se deben realizar estudios de sensibilidad de los parámetros en condición normal, debido a las condiciones de reproducibilidad de los ensayos.

\section{AGRADECIMIENTOS}

Los autores agradecen a COLCIENCIAS en Colombia y al Ministerio de Ciencia e Innovación del gobierno Español, por su apoyo en el desarrollo de este trabajo a través del proyecto DPI200805890 .

\section{REFERENCIAS}

Bjerkan E., Høidalen H. y Moreau O., FRA sensitivity analysis using high frequency modeling of power transformers based on the finite element method, Proceedings of the XIVth International Symposium on High Voltage Engineering, Tsinghua University, Beijing, China, Agosto, (2005).

CIGRE Technical Brochure 342, Mechanical condition assessment of Transformer windings using Frequency Response Analysis (FRA), Working Group A2.26 April (2008).

DL/T 911. The Electric Power Industry Standard of People's Republic of China. Document 15182. Frequency Response Analysis on Winding Deformation of Power Transformers. China (2004).

Dick, E. P. y Erven. C. C., Transformer Diagnostic Testing by Frequency Response Analysis, IEEE/PAS-97. No. 6. pp.2144-2153, (1978).

Florkowski M. y Furgal J., Modelling of winding failures identification using the frequency response analysis (FRA) method, Electric Power Systems Research 79, pag. 1069-1075,(2009).

Herszterg K., Martins H. y Carneiro S., Analytical approach of frequency response through a mathematical model of transformer windings, Proceedings of the XIVth International Symposium on High Voltage Engineering, Tsinghua University, Beijing, China, August, (2005).

IEEE PC57.149тм/D8. Draft trial use, Guide for the Application and Interpretation of Frequency Response Analysis for Oil Immersed Transformers, October (2009).

IEC 14/626/CD Committee Draft, IEC 60076-18 Ed.1: Power transformers - Part 18: Measurement of frequency response, November (2009).

Jayasinghe, J.A.S.B., Wang, Z.D., Jarman, P.N. y A.W. Darwin, Winding Movement in Power Transformers: A Comparison of FRA Measurement Connection Methods, IEEE Transactions on Dielectrics and Electrical Insulation, Vol. 13, No. 6; December (2006).

Pleite J., Olias E., Barrado A., Lazaro A. y Vazquez J., Transformer Modelling for FRA Tecniques, IEEE-PES Transmission and Distribution Conference and Exhibition, Asia Pacific (2002). 
Pleite, J., "Herramienta de Modelado para el Mantenimiento Predictivo de Transformadores basado en el Análisis de la Respuesta en Frecuencia", Documento Ph.D, Universidad Carlos III de Madrid, Madrid (2000).

Pong, L., Modeling a transformer with Doble M series instruments, Doble Engineering Company. Vol. TX 14 (2004).

Rahimpour, E., Christian J., Feser, K. y Mohseni H., Transfer function method to diagnose axial displacement and radial deformation of transformer windings, Power Delivery, IEEE Transactions, Vol. 18, Issue:2, Pages: 493-505, April (2003).

Ryder S.A., Diagnosing Transformer Faults Using Frequency Response Analysis, IEEE Electrical Insulation Magazine, Vol. 19. No 2, April (2003). 
